\title{
Pandora's Problem with Nonobligatory Inspection
}

\author{
HEDYEH BEYHAGHI, Cornell University \\ ROBERT KLEINBERG*, Cornell University
}

\begin{abstract}
Martin Weitzman's "Pandora's problem" furnishes the mathematical basis for optimal search theory in economics. Nearly 40 years later, Laura Doval introduced a version of the problem in which the searcher is not obligated to pay the cost of inspecting an alternative's value before selecting it. Unlike the original Pandora's problem, the version with nonobligatory inspection cannot be solved optimally by any simple ranking-based policy, and it is unknown whether there exists any polynomial-time algorithm to compute the optimal policy This motivates the study of approximately optimal policies that are simple and computationally efficient. In this work we provide the first non-trivial approximation guarantees for this problem. We introduce a family of "committing policies" such that it is computationally easy to find and implement the optimal committing policy. We prove that the optimal committing policy is guaranteed to approximate the fully optimal policy within a $1-\frac{1}{e}=0.63 \ldots$ factor, and for the special case of two boxes we improve this factor to $4 / 5$ and show that this approximation is tight for the class of committing policies. ${ }^{1}$
\end{abstract}

\section{ACM Reference Format:}

Hedyeh Beyhaghi and Robert Kleinberg. 2019. Pandora's Problem with Nonobligatory Inspection. In ACM EC '19: ACM Conference on Economics and Computation (EC '19), fune 24-28, 2019, Phoenix, AZ, USA. ACM, New York, NY, USA, 2 pages. https://doi.org/10.1145/3328526.3329626

Pandora's problem, introduced in 1979 by Martin Weitzman [4], furnishes the mathematical basis for optimal search theory in economics. The basic elements of the problem are as follows. A searcher is allowed to select one of $n$ closed boxes containing independent random prizes sampled from known (not necessarily identical) distributions. The searcher chooses a sequence of operations, each of which is either opening a box or selecting the box. Opening a box has an associated cost and results in learning the value of the prize contained inside. A box can only be selected after it is opened, and when this happens the searcher receives the value of the prize contained therein and the process ends immediately. The searcher's goal is to design an adaptive policy (i.e., a choice of which operation to perform next, for every possible past history of operations and their outcomes) to maximize the expectation of the prize selected, minus the sum of the inspection costs accrued while opening boxes.

A key assumption in Pandora's problem is that the searcher must open a box, and suffer the attendant cost, before selecting it. This assumption limits the applicability of the model. Given that the value of the prize inside a box is drawn from a distribution known in advance, in many cases it may be more advantageous to select a box without paying to inspect its contents. For example, when using Pandora's problem to model a firm searching for an employee to hire, boxes represent job candidates. The cost of opening a box represents the cost to the firm of undertaking a process, such as an interview or internship, to assess the value of hiring a candidate. If the evidence of a

\footnotetext{
*Supported in part by NSF grant CCF-1512964.

${ }^{1} \mathrm{~A}$ complete version of the paper can be found at https://arxiv.org/abs/1905.01428.
}

Permission to make digital or hard copies of part or all of this work for personal or classroom use is granted without fee provided that copies are not made or distributed for profit or commercial advantage and that copies bear this notice and the full citation on the first page. Copyrights for third-party components of this work must be honored. For all other uses, contact the owner/author(s).

EC '19, fune 24-28, 2019, Phoenix, AZ, USA

(C) 2019 Copyright held by the owner/author(s).

ACM ISBN 978-1-4503-6792-9/19/06.

https://doi.org/10.1145/3328526.3329626 
candidate's promise is sufficiently strong a priori, it may be realistic to assume that the firm is willing to hire him or her directly, skipping the costly evaluation process. This motivates a version of Pandora's problem in which a box may be selected without opening it, if the searcher so desires. Laura Doval [2] introduced this variant of optimal search and identified sufficient conditions for the optimal policy to have a simple structure. She also presented examples illustrating that in general, the optimal policy is unlikely to have any simple description. This motivates the study of approximately optimal policies that are computationally efficient, structurally simple, or both. Our work initiates this study.

We define a committing policy to be one that, before it opens any boxes, must pre-commit to a partition of the $n$ boxes into a set of boxes that will never be opened and a set that will never be selected without first being opened; in addition it pre-commits to an order in which the boxes in the latter set will be opened. Such a policy is almost non-adaptive; the only way in which it may adjust its behavior in response to information revealed during the search process is that it may terminate the search early. In this sense, questions about the ability of committing policies to approximate the optimal adaptive policy are akin to questions about adaptivity gaps in stochastic optimization [1,3].

The foregoing discussion inspires two interrelated questions. For which values of $\alpha$ is there a polynomial-time algorithm that $\alpha$-approximates the optimal adaptive policy? What is the adaptivity gap, i.e. the worst-case ratio between the value of the optimal committing policy and that of the optimal adaptive policy? We show that for the general case of Pandora's problem with nonobligatory inspection, there is a polynomial-time algorithm to identify the optimal committing policy, and this policy always attains at least $1-\frac{1}{e}=0.63 \ldots$ fraction of the optimal policy's value. For the case of two boxes we show that the adaptivity gap is never less than $\frac{4}{5}$, and that this bound is tight. The main question left open by our work is whether the factor of $1-\frac{1}{e}$ for the general case of $n$ boxes can be improved. We conjecture that the answer is yes. In fact, we believe it is plausible that the adaptivity gap is never less than $\frac{4}{5}$, even when the number of boxes is greater than 2 .

\section{REFERENCES}

[1] Arash Asadpour and Hamid Nazerzadeh. 2015. Maximizing stochastic monotone submodular functions. Management Science 62, 8 (2015), 2374-2391.

[2] Laura Doval. 2018. Whether or not to open Pandora's box. fournal of Economic Theory 175 (2018), 127 - 158 . https: //doi.org/10.1016/j.jet.2018.01.005

[3] Anupam Gupta, Viswanath Nagarajan, and Sahil Singla. 2017. Adaptivity gaps for stochastic probing: Submodular and XOS functions. In Proc. 28th Annual ACM-SIAM Symposium on Discrete Algorithms (SODA). SIAM, 1688-1702.

[4] Martin L. Weitzman. 1979. Optimal Search for the Best Alternative. Econometrica 47, 3 (1979), 641-654. 